\title{
Detection and Molecular Characterization of Circulating Tumor Cells (CTCs) in Patient with Metastatic Melanoma: A Potential Application of Liquid Biopsy
}

\section{Suresh M Kumar*}

Department of Science, Engineering and Technology, The Pennsylvania State University, West Harrisburg, USA

\begin{abstract}
Melanoma specific anti NG2 antibody conjugated with iron oxide nanoparticles that have been developed to isolate, detect, and culture CTCs from melanoma xenograft models and blood from patients with metastatic melanoma. The enrichment process involved, lysis of RBC from blood samples using RBC lysis buffer enhances the cancer cell recovery by immunomagnetic labeling and separation. Efficient cell capture was validated using fluorescently labeled cancer cells spiked into healthy human blood and clinical utility was demonstrated in specimens from patient with metastatic melanoma. The spiking experiment results with greater than $70 \%$ recovery in four sets of experimental. Spontaneous metastasis melanoma model, found that the number of CTCs increased during the tumor progression and correlated to lymphatic as well as lung metastasis development in vivo. CTCs were detected in 6 out of 7 patients' blood samples that contained metastatic melanoma. The CTCs were positive for melanoma specific molecular markers such as S100, HMB45, MelaA, MITF and the CTCs were positive for anti NG2-Q-dot and pERK2-Q-dot staining. The BRAFv600e gene expression pattern resulted that the CTCs were expressed BRAF ${ }^{\mathrm{V} 600 \mathrm{e}}$ similar to the control melanoma cells. The iron oxide antibody nanoparticle isolation method is a highly reliable and cost effective detection method of CTCs from patients with metastatic melanoma and CTCs cells can be culture for additional analytical studies or potential drug sensitivity testing.
\end{abstract}

Keywords: Melanoma; CTCs; Iron oxide; Antibody; Metastasis

Abbreviations: CTCs: Circulating Tumor Cells; IO: Iron Oxide; MITF: Microphthalmia-associated transcription factor; Q-dot: Quantum-dot

\section{Introduction}

Circulating tumor cells (CTCs) are cancer cells shed from primary and secondary metastatic tumor sites which are then transported through the blood stream to distant vital organs. These cells are then mixed with the other components of the blood making their isolation and characterization technologically difficult due to their rarity when compared to the frequency of other components of the blood. Detection and enumeration methods of CTCs from peripheral blood have been examined in many types of metastatic cancer such as breast [1-3], prostate [4-6] and colorectal [7-9] cancers in addition to melanoma [10-13]. The amount of CTCs present in the blood stream of cancer patients is extremely small and it has been estimated for every CTC in the blood of a cancer patient there are $10^{4}-10^{7}$ normal blood cells [14]. CTCs are highly heterogeneous, and their molecular characterizations are important not only to confirm their malignant origin, but also to follow their immune-phenotypic changes during tumor progression. As a result, the isolation and analysis of CTCs may provide new insight into reveal the dynamic process of tumor metastasis during tumor progression. The detection of CTCs and assessment of micro metastasis are important for prognostic and therapeutic implications [15]. The detection of CTCs in patients ultimately results in the increased efficacy of drug treatment and biomarker for prognosis assessment.

The analytical methods of detection and purification of CTCs, include separation based on cell size, fast scanning cytometry, and immunomagnetic bead methods [16]. However, most of the CTC detection studies performed based upon immune-affinity and density gradient centrifugation enrichment methods using antibodies against surface antigens. The efficacy of these method, in terms of analytic capability, is limited due to the fact that isolated CTCs become dormant and lose cell viability or metastatic potential $[17,18]$.
Numerous studies have shown that CTCs can be detected by reverse transcriptase polymerase chain reaction (RT-PCR) targeting specific mRNA gene expression in cancer cell types $[19,20]$. Melanoma markers such as tyrosinase, MUC18, MITF glycoprotein gp100/pmel17, and MART1/mela.A were all used as molecular markers in the detection of melanoma CTCs. The sensitivity of this molecular technique has been well demonstrated. However, the quality control for diagnostic use remains problematic because of the false positive and false negative results [21]. Advanced molecular techniques such as quantitative real time polymerase chain reaction (qRT-PCR) and microarray gene expression showed increased detection sensitivity as well as improved quantitative analysis of CTCs isolated from melanoma patients [22]. Several qRT-PCR based multi marker assays such as tyrosinase, p97, MUC16, melanA/MART1, MAGE3 and/or gp100/pMel-17 have been reported for CTC detection in melanoma patient blood samples [22-24].

The presence of tumor cells in blood is predictive of short survival in several cancers and their isolation and characterization can guide towards the use of more effective treatment. The CTCs are re highly heterogeneous in nature and number of clinical research studies has been undergoing the validation of CTCs observation and characterization. In this paper, we demonstrated the separation of circulating tumor cells (CTCs) from in vivo melanoma xenograft

*Corresponding author: Dr. Suresh M Kumar, PhD, Department of Science Engineering and Technology, 777 The Pennsylvania State University, West Harrisburg Drive, Middletown, PA 17057, USA, Tel: +112152984229; E-mail: smk31@psu.edu

Received May 26, 2016; Accepted June 10, 2016; Published June 17, 2016

Citation: Kumar SM (2016) Detection and Molecular Characterization of Circulating Tumor Cells (CTCs) in Patient with Metastatic Melanoma: A Potential Application of Liquid Biopsy. Clin Med Biochem Open Access 2: 119. doi:10.4172/24712663.1000119

Copyright: () 2016 Kumar SM. This is an open-access article distributed under the terms of the Creative Commons Attribution License, which permits unrestricted use, distribution, and reproduction in any medium, provided the original author and source are credited. 
models and metastatic melanoma patient blood samples using a melanoma specific anti NG2 (9.2.27) antibody conjugated with ironoxide nanoparticles under a magnetic field. This cells surface antigen is highly expressed on melanoma cells. The isolated rare CTCs cells were able to proliferate under in vitro conditions.

\section{Materials and Methods}

\section{Cell lines}

The B16-F10 murine melanoma, C8161, 1205Lu, and $451 \mathrm{Lu}$ A375M melanoma cell lines were cultured in DMEM containing $10 \%$ fetal bovine serum. Primary human melanoma cells from melanoma patient's tumor tissues by previously published protocol [25]. Briefly, the tissue samples were washed with PBS and the tissues were mechanically chopped into small pieces followed by enzymatic digestion using $20 \mu \mathrm{g} / \mathrm{mL}$ concentration of collagenase type 1 solution and incubation of the tissues at $37^{\circ} \mathrm{C}$ for one hour. The cells were sieved through a cell strainer $(100 \mu \mathrm{m})$ size and the cells were washed with PBS and cultured in DMEM supplemented with 10\% FBS and Penn/ strep solution. For GFP lent viral production, a GFP plenti viral vector was co-transfected into $293 \mathrm{~T}$ cells with a packing vector (pCMV $\Delta 8.2 \mathrm{durp}$ and pCMV, VSVG), (generous gift from Dr. Xiaowei Xu at university of Pennsylvania), and the viral supernatants were collected at 48 and 72 $\mathrm{h}$ post transfection [26].

\section{Patient and blood sample collection}

After the informed consent for participant in this institutional review board-approved study, each patient's blood $(5-10 \mathrm{~mL})$ was collected in EDTA tubes at the time of surgery in the university hospital and processed within $2 \mathrm{hrs}$. The research operators were blinded to all clinical and histopathological correlative information during the sampling analysis process.

\section{Red blood cell lysis}

In the clinical blood samples from cancer patients and healthy donors, the red blood cells were removed by applying a RBC lysis buffer ( $154 \mathrm{mM} \mathrm{NH}_{4} \mathrm{Cl}, 10 \mathrm{mM} \mathrm{KHCO}_{3}$ and $0.1 \mathrm{mM}$ EDTA). Briefly, $1 \mathrm{~mL}$ of a blood sample was added into $25 \mathrm{~mL}$ of pre-warmed RBC lysis buffer and incubated for $5 \mathrm{~min}$ in a shaking condition at room temperature. The sample was then centrifuged at $300 \mathrm{~g}$ for $5 \mathrm{~min}$. The cell pellets were washed two times with pre-warmed PBS and the cells were suspended in $1 \mathrm{~mL}$ DMEM. The cell suspension was then ready for magnetic labeling.

\section{Immunomagnetic labeling and magnetic separation}

To perform immunomagnetic labeling, we have used a carboxyl magnetic iron oxide nanocrystal conjugation kit. The IO np-Ab conjugation was performed according to the manufacturer'sinstructions (Ocean Nano Tech, Springdale, AR). Briefly, $200 \mu \mathrm{L}$ of magnetic iron oxide nanoparticles and $200 \mu \mathrm{L}$ of activation buffer were aliquoted in a sterile eppendorf tube and $100 \mu \mathrm{L}$ of EDAC/NHS was added then incubated for $10 \mathrm{~min}$ with continuous mixing at room temperature. $500 \mu \mathrm{L}$ of anti NG2 (9.2.27) purified antibody and $500 \mu \mathrm{L}$ of coupling buffer were added into the tube and mixed well then incubated for 2 hours with continuous mixing. Next, $10 \mu \mathrm{L}$ of quenching solution was combined with the above mentioned reaction mixture and incubated for another $10 \mathrm{~min}$ with continuous shaking. The reaction mixture was then transferred into a plastic cuvette and $1 \times$ wash/storage buffer was added. The reaction mixture in the cuvette was then placed into the Super Mag Separator ${ }^{\mathrm{TM}}$ magnetic separator (Ocean Nano Tech, Springdale, AR) separate the magnetic iron oxide nanoparticles at $4^{\circ} \mathrm{C}$ for 4 hrs. The separated iron oxide- anti NG2 [9.2.27] antibody complex was washed with $3 \mathrm{~mL}$ of $1 \times$ wash buffer and stored in $1 \mathrm{~mL}$ of $1 \times$ wash/storage buffer. To perform cell separation $50 \mu \mathrm{L}$ of iron oxide- 9.2.27 antibody conjugated nanoparticles was incubated with 1 $\mathrm{mL}$ of RBC lysed blood sample for $2 \mathrm{hrs}$ at room temperature and the immunolabeled cells were separated by using a Super Mag Separator ${ }^{\mathrm{TM}}$ magnetic separator for $2 \mathrm{hrs}$. The magnetically separated cells were washed with 1x PBS and the cells were then resuspended in DMEM supplemented with $10 \%$ FBS and Penn/strep solution then plated in a 24 well plate and incubated in $\mathrm{CO}_{2}$ incubator at $37^{\circ} \mathrm{C}$. The media was changed after $24 \mathrm{hrs}$ with tumor fibroblast condition media with $10 \%$ DMEM (1:1 ratio).

\section{Anti NG2 [9.2.27] antibody production}

The murine hybridoma cells secrete an IgG monoclonal antibody against a $240 \mathrm{kD}$ human melanoma surface glycoprotein. The anti NG2 hybridoma cells were grown in a T75 flask containing filter sterilized DMEM with $4 \mathrm{mM} \mathrm{L}$-glutamine and $1.5 \mathrm{~g} / \mathrm{L}$ sodium bicarbonate supplemented with $10 \%$ fetal bovine serum. The culture was maintained at $37^{\circ} \mathrm{C}$ in $5 \% \mathrm{CO}_{2}$ incubator. To make anti NG2 antibody, the viable hybridoma cells were seeded into a flask containing a combination of $\mathrm{BD}$ cell ${ }^{\mathrm{TM}} \mathrm{M} \mathrm{Ab}$ medium animal component free media (BD biosciences CA and DMEM supplemented with 10\% FBS in the ratio of 50:50. The monoclonal antibody containing media was collected according to the hybridoma culture method and the concentration of antibody was determined by standard protein estimation methods.

\section{In vivo studies}

All animal studies were performed under a protocol approved by the IACUC at the Pennsylvania State University. Green Fluorescent Protein (GFP) tagged murine melanoma cells B16-F10 $\left(2 \times 10^{5}\right.$ cells per injection) and metastatic human melanoma cells $451 \mathrm{Lu}, 1205 \mathrm{Lu}$ and $\mathrm{A} 375 \mathrm{M}\left(2.5 \times 10^{6}\right)$ were injected into the flank region of the intradermal layer of C57B/L6 mice for B16- F10 GFP cells and in athymic nude mice injected $451 \mathrm{Lu}, 1205 \mathrm{Lu}$ and $\mathrm{A} 375 \mathrm{M}$ cells. The tumor challenging experiments were preformed observed for 21 to 24 days and measure tumor size, collect blood for CTC analysis, dissect out the lymphnodes and other internal organs at each time points.

\section{Q-Dot staining, immunocytochemistry, western blotting and qRT-PCR}

The melanoma and CTC cells were grown on eight well chamber slides and the monolayer cells were fixed with $4 \%$ paraformaldehyde and stained with primary antibodies specific for MITF (Neomarker, Carlsebad, CA) S100, HMB45, Mela-A, CD45 (Dako North America, INC Carpinteria, CA) and anti-NG2 (9.2.27) antibodies. The cells were incubated with primary antibodies overnight at $4^{\circ} \mathrm{C}$. After washings, the cells were incubated with appropriate Alexa fluor secondary antibodies (Life Technologies, Grand Island, NY) and mounted on DAPI containing mounting media (Vector laboratories Inc., Burlingame, CA, USA). Q-dot staining was performed according to the manufactures protocol (Life Technologies, Grand Island, NY), for western blot analysis, a monolayer of melanoma cells and the CTC cells were washed with ice- cold PBS and lysed in RIPA buffer or lamella buffer to analyze total protein content. Fifty microgram of proteins were separated in Nu-PAGE4-15\% Bis-Tris Gel (Life Technologies, Grand Island, NY) and transferred to polyvinylidene difluoride (PVDF) membrane (Life Sciences). The membrane was blocked and then incubated with antibodies against human MITF (Neomarker, Fromont, CA) and $\alpha$-Enolase (Santa Cruz, CA) in 5\% milk TBST buffer (150 mmol/L Tris-HCL (pH 8.0), $150 \mathrm{mmol} / \mathrm{l} \mathrm{NaCl}$ and 0.01\% Tween 
20 and $5 \%$ non-fat milk. The membranes were washed thrice with wash buffer for $5 \mathrm{~min}$ and incubated with horseradish peroxidase-conjugated secondary antibody and washed again before being processed with chemiluminescence reagents (ECL Western Blotting Detection System; Pierce, Rockford, IL, USA). Quantitative RT-PCR was performed according to previously published protocol [26].

\section{Statistical analyses}

The data represent mean \pm S.E.M values. The differences between two experimental groups were determined using Student's $t$-test. A two-tailed value of $P<0.05$ was considered statistically significant.

\section{Results}

\section{Characterization of Iron Oxide (IO) nanoparticles}

Carboxyl magnetic iron oxide (IO) nanocrystal conjugation kit was purchased from Ocean Nano Tech (Springdale, AR). The IO nanocrystals were conjugated with melanoma specific antibody anti NG2 (9.2.27) according to the manufacture's protocol. The size and charge of the anti NG2- Iron Oxide nanoparticles were measured by zeta analyzer (Malvern Zetasizer- nanoseries, Malvern Instruments limited, Worcestershire, UK). The particle size observed for the antibody conjugated IO particle was increased (Figure $1 \mathrm{~A}$ and $1 \mathrm{~B}$ ) along with an increase in the zeta potential of the IO-Ab nanoparticles from - 36.8 $\mathrm{mV}$ to $-35.6 \mathrm{mV}$. The less negative zeta potential can be attributed to the surface charge shielding of the carboxyl groups on the surface of the unconjugated iron oxide [IO] nanoparticles after conjugated to the amine groups of the antibodies. The increase in size may be attributed to the conjugation of the antibodies plus the water of hydration on the conjugated surface. The migration of the conjugated IO-antiNG2 nanocrystals observed during gel electrophoresis in a $1 \%$ agarose gel towards the positive pole was much slower than the unconjugated IO nanocrystals. This can be attributed to the increased molecular weight of the IO-antiNG2 complex and less negative zeta potential (Figure 1C). Since the capture and conjugation of antibody was performed directly from the reaction media, it is important to check the binding affinity of antibody with the iron oxide nanobeads, which was done by resolving the conjugated antibody on a SDS-PAGE gel (data not shown) and the results indicated that both the heavy $(55 \mathrm{KDa})$ and the light chain $(25$ $\mathrm{KDa}$ ) seen in iron oxide conjugated antiNG2 antibody.

\section{Anti NG2 protein expression in melanoma cells}

Anti NG2 (9.2.27) hybridoma cells were cultured in a hybridoma specific culture medium. After obtaining a continuous culture, the monoclonal antibody was isolated from the culture medium and purified by affinity column chromatography. The purified anti NG2 antibodies were the subjected to western blot analysis as well as immunocytochemistry (Figure 2) melanoma cells $(1205 \mathrm{Lu}, 451 \mathrm{Lu}, \mathrm{A} 375 \mathrm{M}$, WM793 and UACC903), and breast cancer cells (MCF-7) used as a negative control. The western blot results showed that melanoma cells were expressed high level of anti NG2 protein (Figure 2A). Furthermore, we have detected this cell surface marker protein in melanoma cells by immunocytochemical staining methods. Three independent observations were performed for the evaluation of cell surface protein. Melanoma Cell C8161 (Figure 2B), and tumor derived melanoma cell lines (data not shown) showed a high intensity of anti NG2 staining.

\section{Spiking melanoma cells into human blood and culture in vitro}

There are certain limitations of CTC isolation methods used to isolate CTCs from the blood of tumor-bearing animals that only permit in situ analysis of collected cells within the separation device. In this method, we demonstrated that melanoma cells spiked into the blood can be isolated and then cultured in vitro. It is important to note that this experiment does not mimic actual model of melanoma metastasis in which cells would have to detach from a primary tumor site and survive circulation through the entire vasculature before being isolated. For the effective capture and detection of CTCs, we used melanomaspecific antibody NG2 conjugated with iron- oxide nanocrystals. We spiked different cell numbers $(1000,500)$ of metastatic melanoma cells (1205Lu-GFP, 451Lu-GFP) and cells derived from metastatic melanoma tissue samples tagged with Green Flourescent Protein (GFP) into fresh healthy human whole blood. The result shown in Figure $2 \mathrm{C}$, the 1205Lu-GFP, 451Lu-GFP, and melanoma tumor cells (data not shown) were successfully captured directly from whole blood and RBC lysed samples. The same amount of cells spiked into the whole blood and RBC lysis samples were seeded in a separate chamber as a positive control. In addition, used breast cancer cell line (MCF7) and human fibroblast (FF10) was used as negative control for testing the binding affinity of Iron oxide conjugated Anti NG2 antibody. The percentage of recovery was calculated according to previously published methods [27]. The percentage of recovery was higher The samples that underwent RBC lysis with percent recoveries of $70 \%$ and $85 \%$ which were substantially higher than the percent recovery values from the whole blood samples (less than 30\%). The captured cells were cultured in 10\% DMEM and successful colony formation and proliferation was observed without any deterioration of cell morphology and viability (Figure 2D). We obtained blood and tumor tissue samples from patients diagnosed with a $\mathrm{BRAF}^{\mathrm{V} 600 \mathrm{E}}$-mutant and $\mathrm{BRAF}^{\mathrm{WT}}$ cutaneous melanoma. The BRAF mutation appears to have critical role in tumor initiation and melanoma progression. We investigated the gene expression pattern of BRAFWT [Sbcl2, WM3211] and BRAF ${ }^{\mathrm{V} 600 \mathrm{E}}$ [WM793, 1205Lu] melanoma cell lines and CTCs isolated from melanoma patient's blood samples. Our result demonstrated that, the expression of $\mathrm{BRAF}^{\mathrm{V} 600 \mathrm{E}}$ expression was homologous in melanoma primary cell lines and in CTCs of patients with metastatic melanoma (Figure 2E). However, the melanocyte and $\mathrm{BRAF}^{\mathrm{WT}}$ cell lines, the $\mathrm{BRAF}^{\mathrm{V} 600 \mathrm{E}} \mathrm{mRNA}$ expression remain same or less expressed.

\section{Assessment of spontaneous metastasis}

Here we report that syngenic model of spontaneous metastatisis of B16-F10 mouse melanoma cells in C57BL/6 and highly metastatic melanoma cell $(451 \mathrm{Lu}, 1205 \mathrm{Lu}$ and $\mathrm{A} 375 \mathrm{M})$ in athymicnude mice with a very high frequency that mimics clinical metastatic melanoma. Two hundred thousand of B16-GFP mouse melanoma cells as well as metastatic human melanoma cells $\left(2.5 \times 10^{6}\right)$ were injected intradermally into the flank region of the skin. Metastatic melanoma is typically first detected by the presence of lymph node metastases and less often by systemic metastases. Lymphatic and visceral metastases were observed in all animals tested. The GFP tagged melanoma cells were observed in all animals at day 7 and an increase in the amount of GFP cells during the tumor progression was apparent. The tumor weight was correlated with the tumor growth time and also the significant number of micro metastases in the lymph nodes. However the appearance of CTCs and lung metastasis was not seen during the early stages of the tumor development in both tumor progression models (Figure $3 \mathrm{~A}$ and $3 \mathrm{~B}$ ). To evaluate the efficacy of anti NG2iron oxide nanocrystals techniques for in vivo CTC capture, we separated circulating tumor cells (CTCs) from the blood of melanoma xenograft models. The blood samples $(\sim 1 \mathrm{ml})$ taken from those animals 2 weeks after tumor cell implantation were mixed with anti NG2 coated magnetic beads and were subjected to magnetic separation. Isolated CTCs were then washed with PBS and plated in 24 
A

$30 \mathrm{~nm}$ IONP only

\begin{tabular}{|c|c|c|c|c|c|}
\hline & & & Mean (mV) & Area $(\%)$ & Width (mV) \\
\hline Zeta Potential (mV): & -36.8 & Peak 1: & -36.8 & 100.0 & 6.44 \\
\hline Zeta Deviation (mV): & 6.44 & Peak 2: & 0.00 & 0.0 & 0.00 \\
\hline onduct ivity $(\mathrm{mS} / \mathrm{cm})$ : & 0.0228 & Peak 3: & 0.00 & 0.0 & 0.00 \\
\hline
\end{tabular}

Result quality : Good
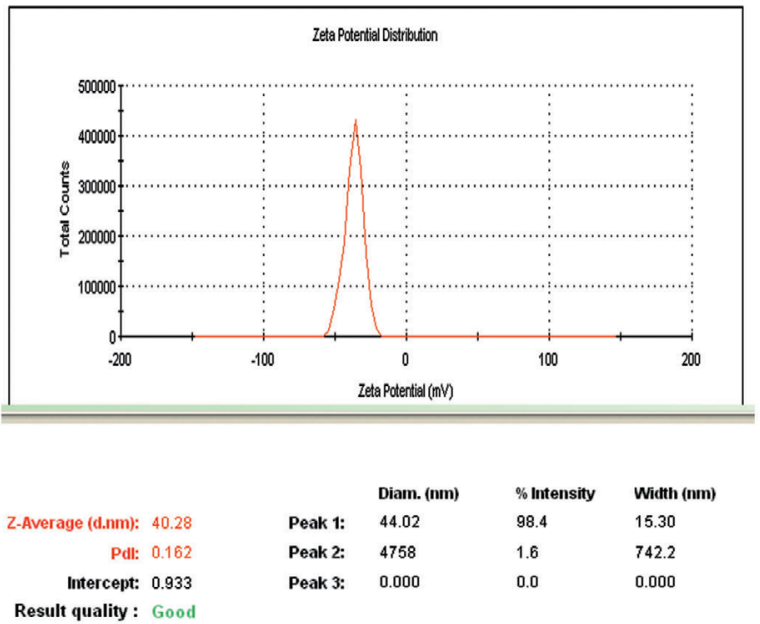

$\mathrm{B}$

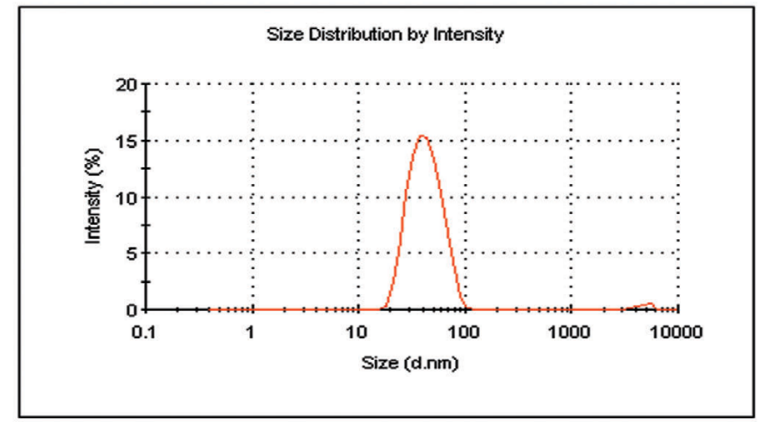

\section{0 nm IONP-Ab}

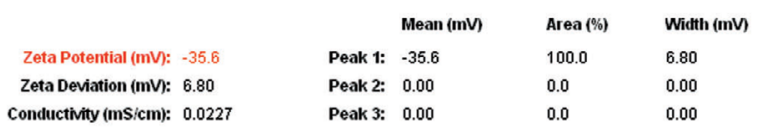

Result quality : Good
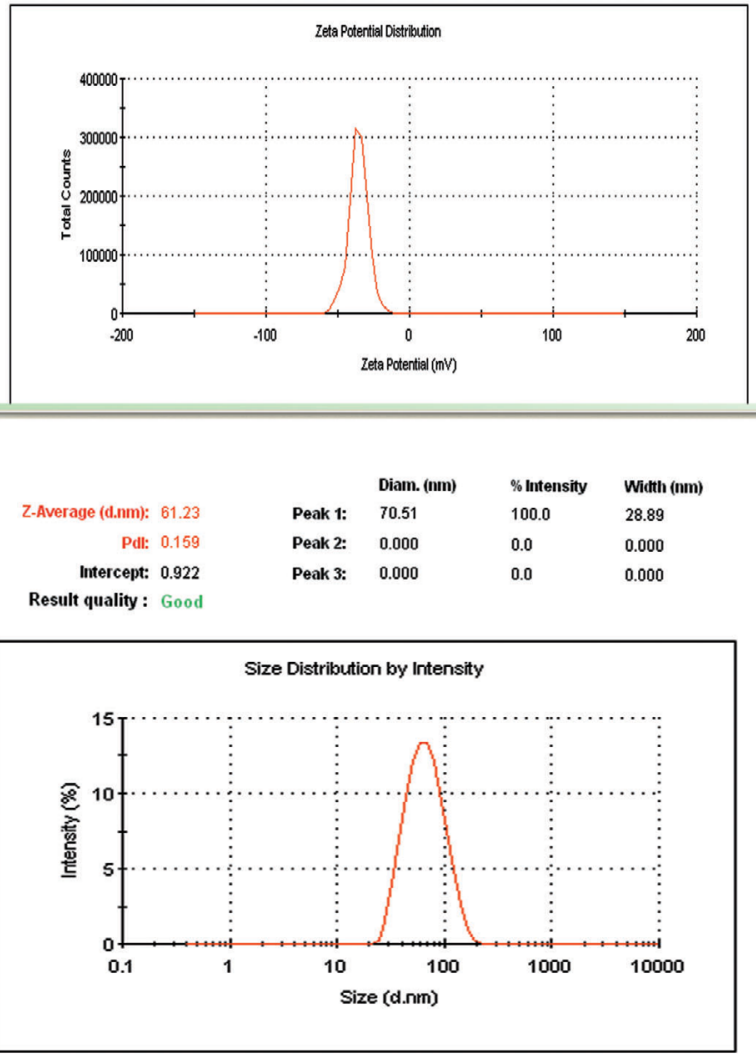

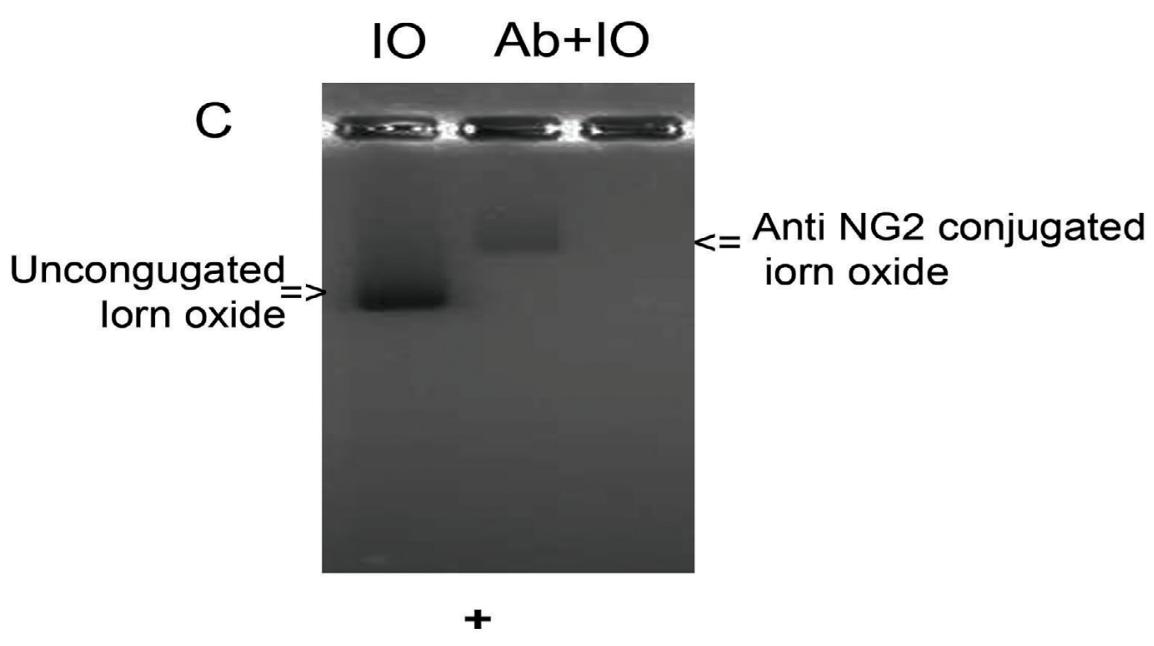

Figure 1: Characterization of Iron Oxide (IO) nanoparticles. A and B) Charge and size of iron oxide conjugated with melanoma specific antibody anti-NG2. The biocompatible water soluble iron nanoparticle size around $40 \mathrm{~nm} \pm 5$ with a zeta potential of $-36.8 \mathrm{mV}$ and after antibody conjugation with size of iron oxide was around $61 \pm 5$ with a zetapotential of $-35.6 \mathrm{mV}$. C) Agarose gel electrophoresis of biocompatible water soluble iron oxide [IO] (on left) and IO-anti NG2 antibody (on right), the electric pole is negative for top and positive for the bottom. 
Citation: Kumar SM (2016) Detection and Molecular Characterization of Circulating Tumor Cells (CTCs) in Patient with Metastatic Melanoma: A Potential Application of Liquid Biopsy. Clin Med Biochem Open Access 2: 119. doi:10.4172/2471-2663.1000119

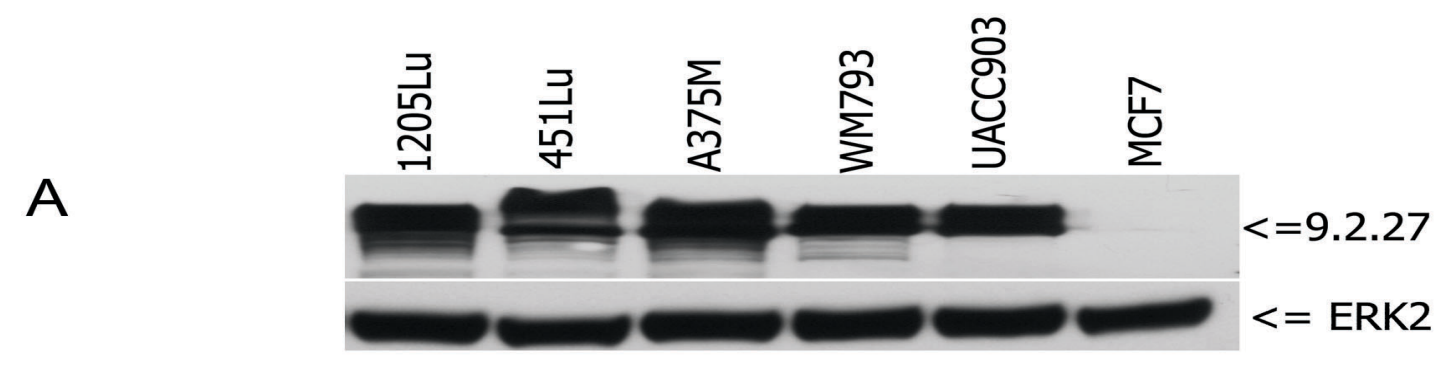

B

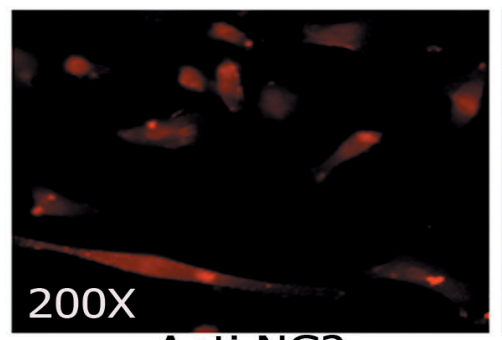

Anti NG2

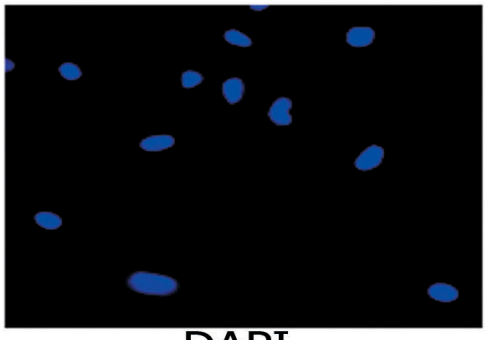

DAPI

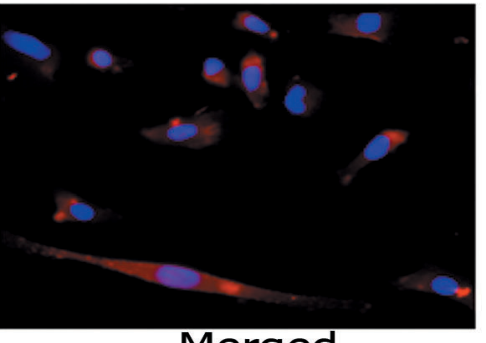

Merged

C

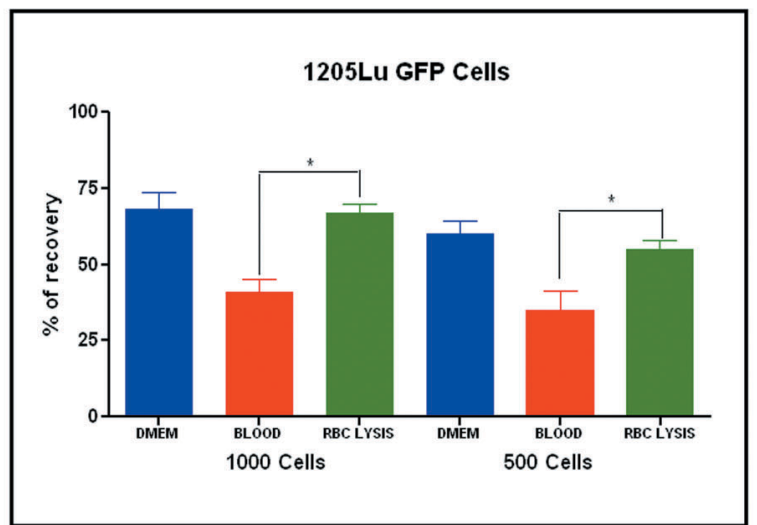

$\mathrm{D}$

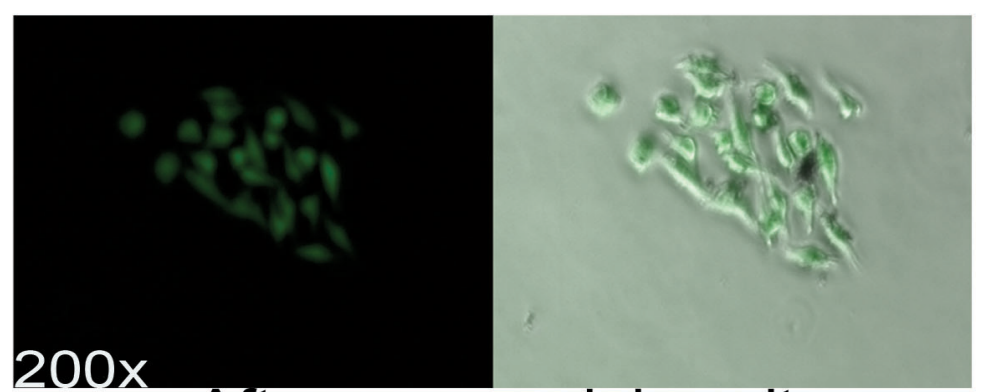

After one week in culture

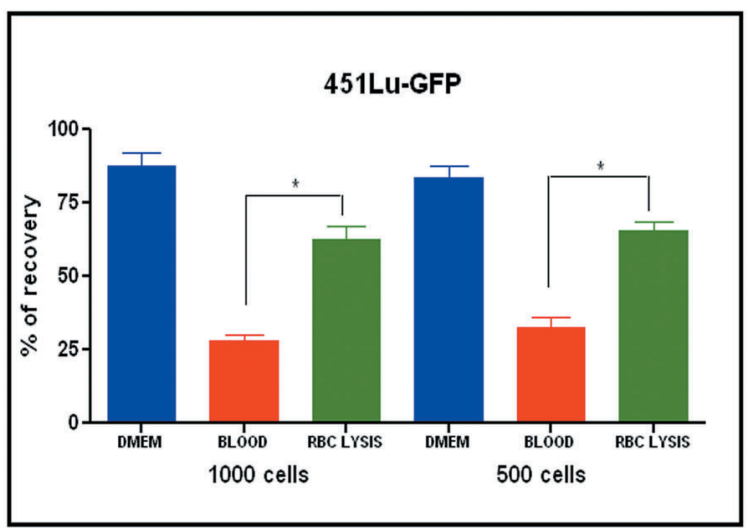

$\mathrm{E}$

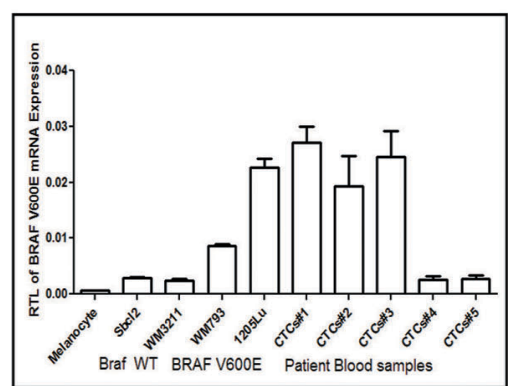

Figure 2: Melanoma cell separation from DMEM and in spiked human blood and RBC lysis buffer. A) Western blot analysis of 9.2 .27 protein expression in metastatic melanoma cell lines. Erk2 antibody used as loading control. B) Immunostaining of anti-NG2 antibody in human melanoma 451Lu GFP cells. GFP over expressing melanoma cells $1205 \mathrm{Lu}$. C) 451Lu cells separated using anti NG2 conjugated iron oxide $(n=3)$. The percentage of recovery rate was significantly high in RBC lysis method. D) Image of melanoma cells recovered from blood spiking using anti NG2 iron oxide nanobeads (Scale 200X). E) Quantitative RT-PCR was performed and showed that BRAFV600E expression level in different melanoma cell lines and melanoma patient CTCs. 

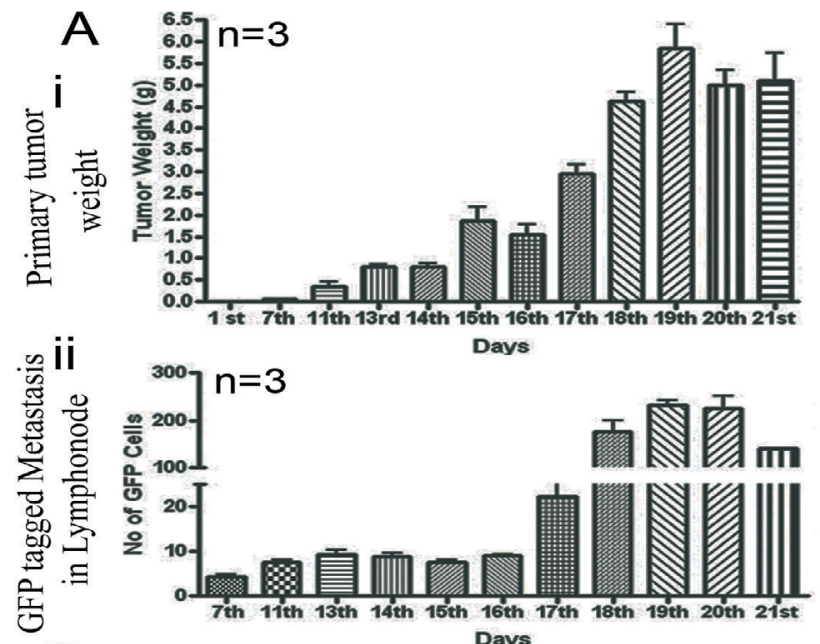

iii
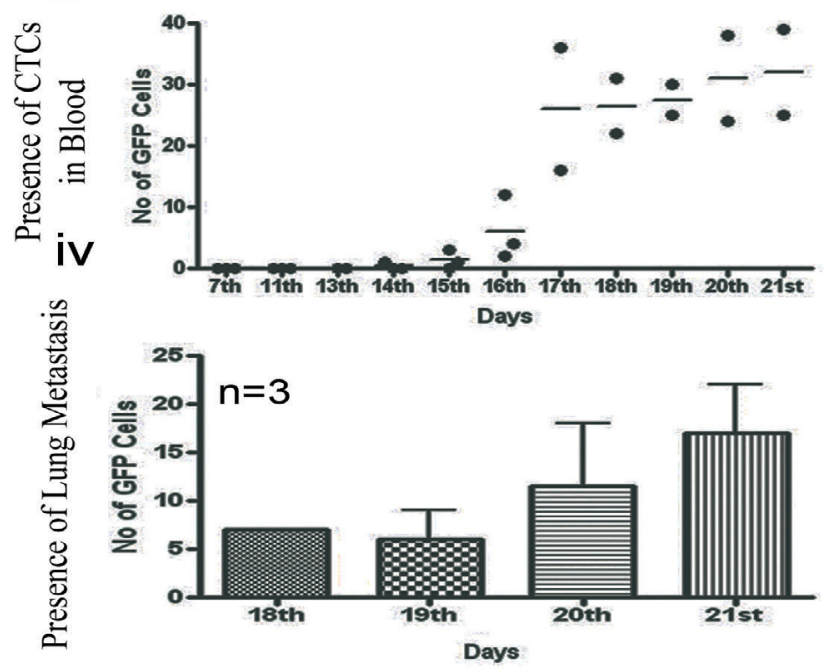

B
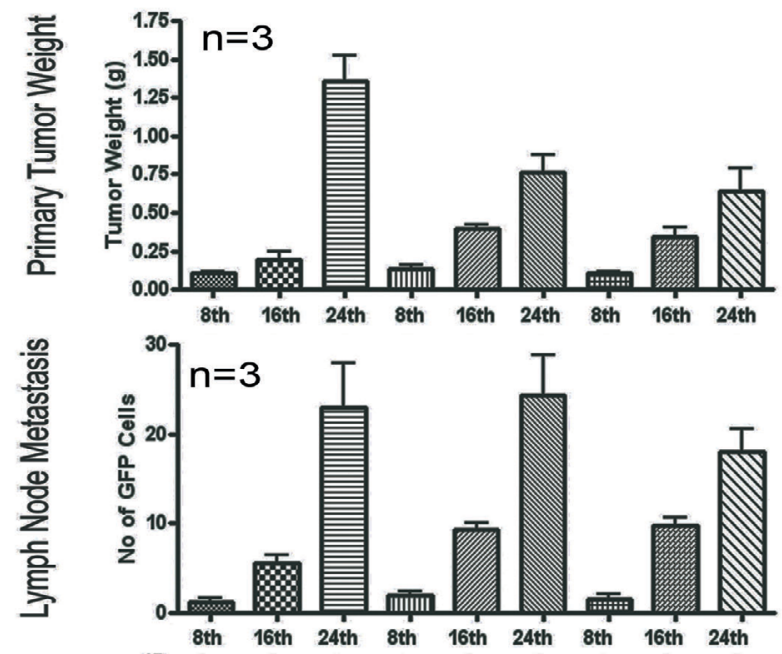

음

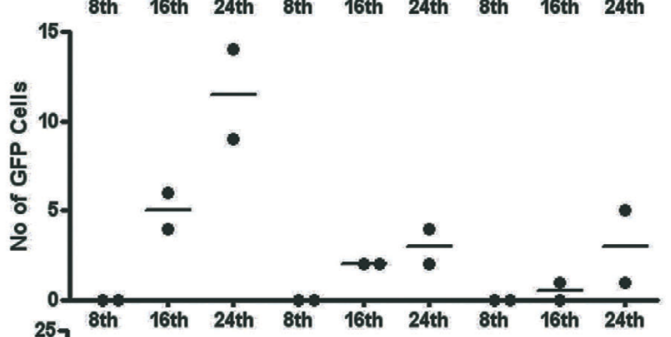

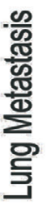

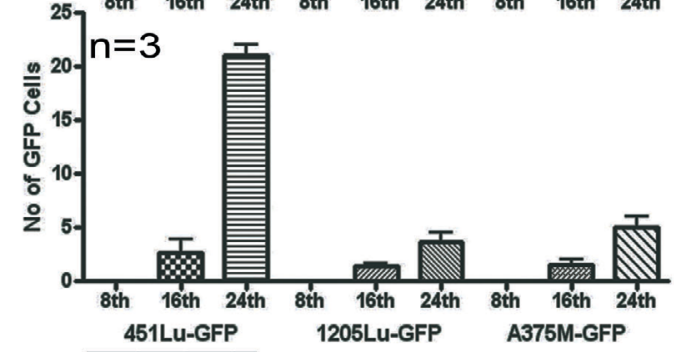

Figure 3: Development of spontaneous metastasis. A) $2 \times 10^{5}$ B16-GFP murine melanoma cells injected intradermally into the flank region of C57BL/6 mice ( $\mathrm{n}=34$ ). i: Tumor weight (gm) of primary tumor after cell injection. ii: Quantification of the frequency of GFP+ fluorescent cell in the lymphnodes during the tumor progression. iii: Circulating tumor cells (CTCs) analysis from blood using anti NG2-iron oxide immunomagnetic separation. iv: Quantification of the frequency of GFP ${ }^{+}$fluorescent cells in the lungs metastasis development. B) Melanoma spontaneous tumor progression and metastasis. Quantification of tumor-derived GFP+ metastatic melanoma cells in lymph node, CTCs and lung metastasis. $2 \times 10^{6} \mathrm{GFP}^{+}$melanoma cells (451Lu, 1205Lu, A375M) injected intradermally into the flank region of the athymic-Foxn1 ${ }^{\text {nu }}$ nude mice ( $\left.\mathrm{n}=18\right)$. i: Tumor weight (gm) of primary tumor measured at the time of sacrifice the mice. ii: Quantification of GFP+ Cells in the lymph nodes during tumor progression. iii: Circulating tumor cells (CTCs) analysis from blood using anti NG2-iron oxide nanocrystal separation. iv: Quantification of the frequency of GFP ${ }^{+}$fluorescent cells in the lungs metastasis development after intradermal injection. All animal experiments were performed according to the guidelines of IACC of Penn State University.

well plates containing DMEM supplemented with 10\% FBS and pen/ strep solution. After $24 \mathrm{hr}$, GFP tagged melanoma cells were counted under a fluorescent microscope. We observed that the CTCs appeared in the blood after the $14-16^{\text {th }}$ day of post cancer cell injection in both xenograft models. The number of CTCs increased during the tumor progression. The CTCs adhered well and proliferated on the culture dishes used (Supplementary Figure 1A). The isolated CTCs remains amephenotypic and genotypic characteristic of the parental cells. These results clearly indicate that rare CTCs isolated from the blood collected from xenograft models retain their viability and proliferation capacity during the transition from in vivo to in vitro conditions.

\section{Capture of CTCs from patient with metastatic melanoma}

Blood samples from six patients in various stages of treatment for metastatic melanoma were processed with anti NG2 conjugated iron- oxide nanoparticle. We also processed blood from a healthy individual as a control $(n=3)$. The captured cells were cultured in regular culture conditions and CTC cells were attached and grown on the surface of the culture dish with the media being changed every two days. After a week, the growth of CTC cells was clearly visible in the dish and the CTCs had morphological features analogous to the pointed spindle shape of melanocytes (Supplementary Figure 1B) and could not detect any CTCs cells in control samples. The CTCs cells were trypsinized and grown in chamber slides for immunocytochemical examination with melanoma specific antibodies such as S100, MITF, HMB45, Mela A and a leukocyte marker CD45 (Figure 4A). Furthermore, we quantified the positively stained CTCs cells against DAPI staining to confirm percentage of melanoma marker staining (Figure 4B). The western blot analysis showed that four out of five CTCs from melanoma patients expressed MITF protein, (Figure 4C). The western blot analysis used 
Citation: Kumar SM (2016) Detection and Molecular Characterization of Circulating Tumor Cells (CTCs) in Patient with Metastatic Melanoma: A Potential Application of Liquid Biopsy. Clin Med Biochem Open Access 2: 119. doi:10.4172/2471-2663.1000119
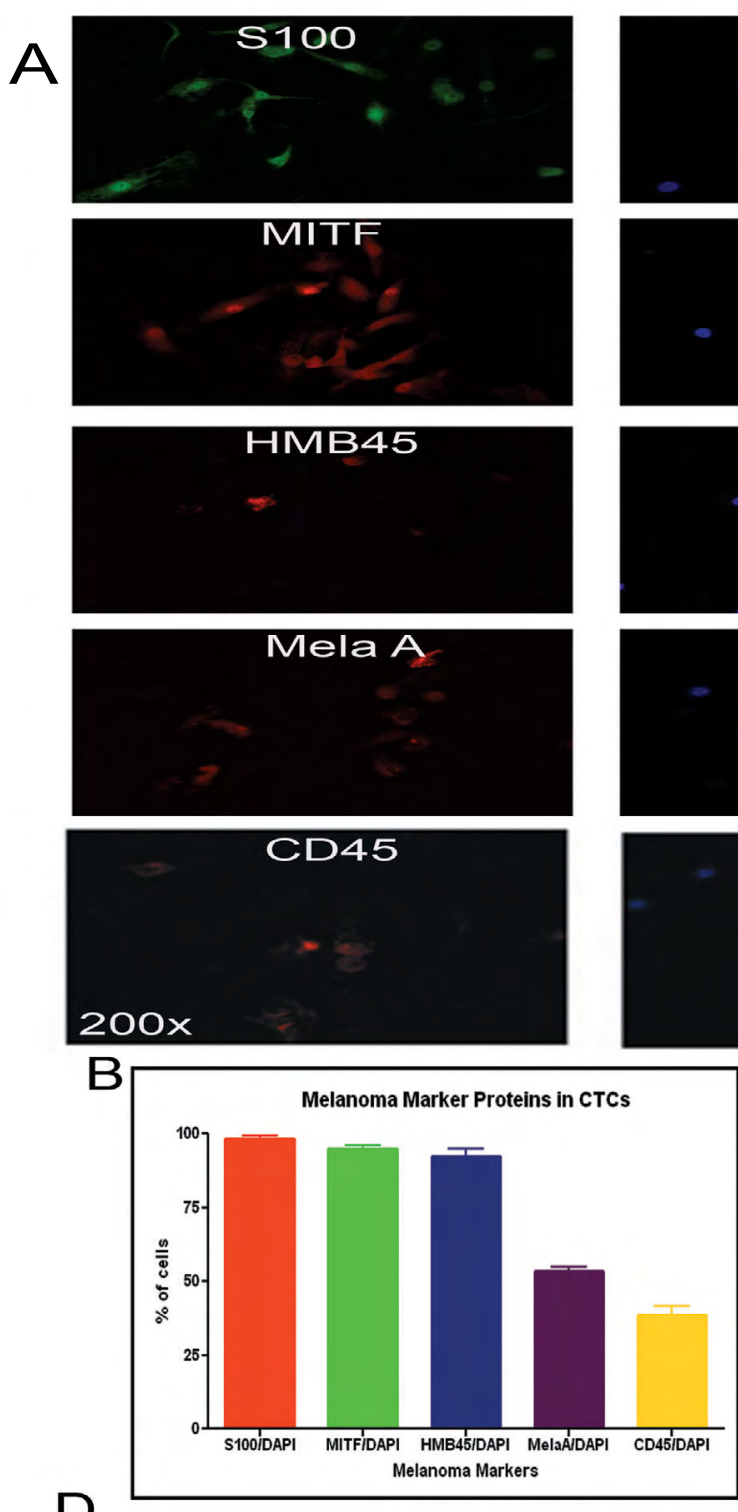

$\mathrm{D}$
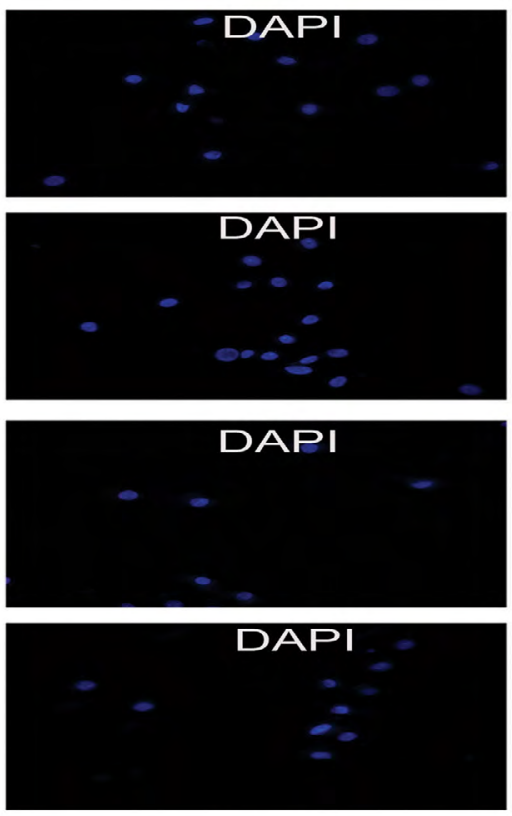

DAPI

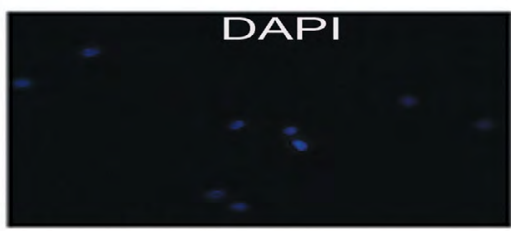

C

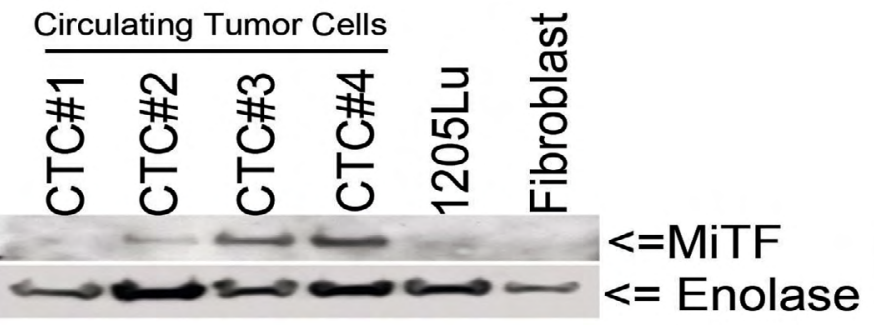

E

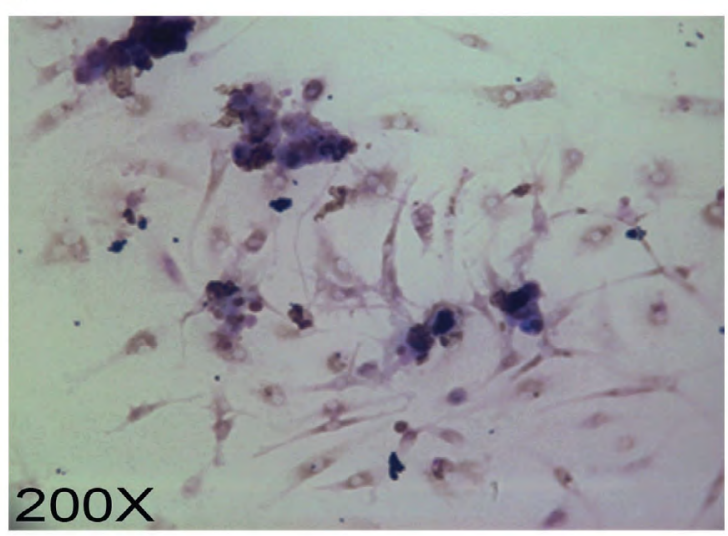

Figure 4: Immuno fluorescent analysis of magnetically isolated CTCs from metastatic melanoma patient blood samples. A) Immunostaining of CTC cells stained with melanoma specific biomarker protein S100, MITF, HMB45 and MelaA. B) Percentage of CTCs cells stained with melanoma against DAPI staining (Scale 200X). C) Western blot analysis of MITF protein expression in CTCs isolated from melanoma metastatic blood sample using anti NG2 conjugated iron oxide nanoparticle. D) Q-DOT staining of CTCs, anti NG2antibody conjugated to q-dot probe (Scale 400X). E) Wright-Gysma staining leukocyte staining of CTCs isolated from melanoma patient blood sample. 
Citation: Kumar SM (2016) Detection and Molecular Characterization of Circulating Tumor Cells (CTCs) in Patient with Metastatic Melanoma: A Potential Application of Liquid Biopsy. Clin Med Biochem Open Access 2: 119. doi:10.4172/2471-2663.1000119

Page 8 of 9

metastatic melanoma cells $1205 \mathrm{Lu}$ as a positive control, fibroblast cells as a negative control, and $a$-enolase as a loading control. The isolated CTCs conformation that had melanoma specific antigen, we performed a Qdot staining with anti NG2 antibody conjugated with a Qdot probe (diameter $=585 \mathrm{~nm} \pm$ ), and a significant level of low abundance expression of melanoma specific antigen in these cells was observed (Figure 4D). The melanoma CTCs were expressing phopshoERK2 after staining with Q-dot probe conjugated with pERK antibody (diameter=605 $\mathrm{nm} \pm$ ) (Supplementary Figure 1C). To further validation, we performed a double stain with S100 and CD45. The result showed that the melanoma specific S100 were co-localized with CD-45 leukocyte markers (Supplementary Figure 1D). Leukocyte contamination is common problem associated with CTCs isolation methods. So we stained the isolated CTC's cells with Wright-Gymsa dye to localize leukocytes contamination in the isolated CTC's cells. It showed that some cells show positive with the staining but some cell are were not stained with Wright-Gymsa dye (Figure 4E).

\section{Discussion}

Tumor cell invasion and extravasation leads to the presence of circulating tumor cells (CTCs) in peripheral blood. However, the CTC counts in patients with cancer would be helpful for analyzing the metastatic mechanism in addition to clinical applications for diagnosis and therapy. In the present study, we have successfully captured and cultured circulating tumor cells (CTCs) from blood samples from melanoma xenograft models and metastatic melanoma patients. The process involved polymer coated $30 \mathrm{~nm}$ iron oxide nanocrytals that were modified with melanoma specific NG2 antibodies. In this study, we demonstrated the separation of CTCs using melanoma specific antibody conjugated with iron oxide nanoparticles (IO-Ab) in an applied magnetic field. The iron oxide nanoparticles were synthesized using a pyrolysis-based method in organic solvent allowing precise control of particle size and crystallinity [28]. The iron oxide nanoparticles which were soluble in organic solvent were modified with polymers to make them water soluble before further modification of the iron oxide nanoparticle with antibody attachment. The successful conjugation of the anti NG2 Ab to the iron oxide nanoparticles was tested using gel electrophoresis. After evaluation of stability, the antibody conjugated IO nanoparticles were used for cancer cell separation from culture media (DMEM) and separation of cancer cells spiked into fresh human blood with and without RBC lysis buffer treatment. The most common problem associated with nanomaterials in biological applications is their compatibility/stability at various biological parameters including, $\mathrm{pH}$, salinity, viscosity and toxicity $[29,30]$. The nanomaterial releases toxic heavy metal irons that cause problems in biological sample separation especially in CTC capturing and the establishment of growth of CTCs in culture conditions. In our studies we successfully captured and grew CTCs from melanoma xenograft models and melanoma patient blood samples without any toxicity problem associated with the iron-oxide nanoparticles.

Recently Clawson et al. reported that CTCs were isolated from metastatic melanoma patient blood samples by simple centrifugation method (OncoQuick column) and characterized by staining with panKRT a melanocytic marker which was aberrantly expressed in melanocyte differentiation [31]. Previous studies were reported that CTCs can be isolated from patient with different types of cancer such as breast cancer using anti HER2 antibody [28] and for prostate cancer using anti PSA antibody conjugated iron oxide nanoparticles [32]. However no studies have been reported with melanoma specific antibody antiNG2 antibody conjugated with iron oxide nanoparticles for CTC isolation and characterization from metastatic melanoma patient blood samples. The accurate quantitative measurement and stability of performance of standards remains challenging. There has been inaccurate assessment as to how many CTCs are released from the primary tumor into the blood daily. The isolation of CTCs is technically challenging due to the rare number ( 1 in $10^{9}$ red blood cells). Multiple batch approaches have been employed to detect CTCs, including cell size based separation [33,34] and fast scanning cytometry [35]. In most of the cases the CTCs are rendered non-viable due the pretreatment process. However the IO based CTC capturing technologies have superiority over other methods, because cell viability remain unchanged and they were proliferate to some extent. While conducting the melanoma cell spiking experiment, we used 6 different cell lines such as UACC903, 1205Lu, 451Lu, A375M, C8161 and B16 mouse melanoma cells which over express GFP protein. The recovery rate was consistent with all the cell lines either in blood or in RBC lysis methods. However the CTCs capturing performance was not same as from cancer patient blood samples. Gertler et al. proposed that CTCs can be distinguished from normal blood cells depends on its physiological properties such as size and difference in cell density, charge, migratory properties and melanocytic granules [36]. Several studies showed that CTCs have significant variation in size difference, and buoyant density compared to the leukocytes [34,37]. These results provide significance outcome in capturing cancer cell lines spiked into blood and further validation required with clinical samples in which heterogeneous nature of cancer cells in the blood. The molecular characterization of CTCs has been conducted in many previous studies. Both qRT-PCR and RT-PCR based methods can detect CTCs and that positive markers are associated with metastatic disease and poor prognosis in melanoma patients [38]. The specificity of these qRT-PCR assays remains controversial because of the indication of false positive results due to the heterogenic nature of mRNA expression of melanoma associated biomarkers in the blood of melanoma patients [39]. The use of immunomagenetic nano bead capture of melanoma cells in our approach exploits the selective expression of melanoma surface antigen in melanoma cells. The CTC isolation techniques rely on antibody-based capture of CTCs which express melanoma specific cell surface markers that are absent from normal leukocytes. Conjugation of antiNG2 (9.2.27) to iron oxide nano beads followed by purification of captured cells through a magnetic field and subsequent staining with melanoma specific antibodies to visualize the expression of melanoma marker protein in captured CTCs. Staining for the leukocyte specific marker CD45 widely used as a negative control to exclude contaminating leukocyte [40]. Wechler et al. (ASCO meeting abstract) isolated CTCs from melanoma patient using Screen Cell method, the isolated CTCs were express S100, MART1/MelaA and HMB45 and their results were consistent with our techniques of iron oxide nano beads conjugated with antiNG2 antibody and cancer cell recovery rates were comparatively higher than Screen Cell system. Our findings revealed the potential clinical usefulness of an immunomagentic nanobead assay for capturing and detecting CTCs in the blood of melanoma patients and melanoma xenograft models. Future studies involving blood analysis and long term followup of patients may be needed for a more detailed assessment of efficacy of drug studies and the clinical utility of this assay.

\section{Acknowledgements}

I thank Dr. Xiaowei Xu, University of Pennsylvania for providing GFP pLenti DNA constructs and Dr. Navaraj Arunasalam, Penn State University Hershey assist taking Q-dot fluorescent images.

\section{References}

1. Lianidou ES, Markou A (2011) Circulating tumor cells as emerging tumor biomarkers in breast cancer. Clin Chem Lab Med 49: 1579-1590.

2. Molloy TJ, Roepman P, Naume B, van't Veer LJ (2012) A prognostic gene expression profile that predicts circulating tumor cell presence in breast cancer patients. PLoS One 7: e32426. 
Citation: Kumar SM (2016) Detection and Molecular Characterization of Circulating Tumor Cells (CTCs) in Patient with Metastatic Melanoma: A Potential Application of Liquid Biopsy. Clin Med Biochem Open Access 2: 119. doi:10.4172/2471-2663.1000119

3. Wang N, Shi L, Li H, Hu Y, Du W, et al. (2012) Detection of circulating tumor cells and tumor stem cells in patients with breast cancer by using flow cytometry: a valuable tool for diagnosis and prognosis evaluation. Tumour Biol 33: 561-569.

4. Magbanua MJ, Sosa EV, Scott JH, Simko J, Collins C, et al. (2012) Isolation and genomic analysis of circulating tumor cells from castration resistant metastatic prostate cancer. BMC Cancer 12: 78

5. Saad F, Pantel K (2012) The current role of circulating tumor cells in the diagnosis and management of bone metastases in advanced prostate cancer. Future Oncol 8: 321-331.

6. Yates DR, Rouprêt M, Drouin SJ, Comperat E, Ricci S, et al. (2012) Quantitative RT-PCR analysis of PSA and prostate-specific membrane antigen mRNA to detect circulating tumor cells improves recurrence-free survival nomogram prediction after radical prostatectomy. Prostate 72: 1382-1388.

7. Gazzaniga P, Gradilone A, Petracca A, Nicolazzo C, Raimondi C, et al. (2010) Molecular markers in circulating tumour cells from metastatic colorectal cancer patients. J Cell Mol Med 14: 2073-2077.

8. Shimada R, linuma H, Akahane T, Horiuchi A, Watanabe T (2012) Prognostic significance of CTCs and CSCs of tumor drainage vein blood in Dukes' stage B and C colorectal cancer patients. Oncol Rep 27: 947-953.

9. Vardakis N, Messaritakis I, Papadaki C, Agoglossakis G, Sfakianaki M, et al (2011) Prognostic significance of the detection of peripheral blood CEACAM 5 mRNA-positive cells by real-time polymerase chain reaction in operable colorectal cancer. Clin Cancer Res 17: 165-173.

10. De Giorgi V, Pinzani P, Salvianti F, Panelos J, Paglierani M, et al. (2010) Application of a filtration- and isolation-by-size technique for the detection of circulating tumor cells in cutaneous melanoma. J Invest Dermatol 130: 2440-2447.

11. Hoshimoto S, Faries MB, Morton DL, Shingai T, Kuo C, et al. (2012) Assessment of prognostic circulating tumor cells in a phase III trial of adjuvant immunotherapy after complete resection of stage IV melanoma. Ann Surg 255 357-362.

12. Ma J, Lin JY, Alloo A, Wilson BJ, Schatton T, et al. (2010) Isolation of tumorigenic circulating melanoma cells. Biochem Biophys Res Commun 402: 711-717.

13. Xu X, Zhong JF (2010) Circulating tumor cells and melanoma progression. $J$ Invest Dermatol 130: 2349-2351.

14. Ross AA, Cooper BW, Lazarus HM, Mackay W, Moss TJ, et al. (1993) Detection and viability of tumor cells in peripheral blood stem cell collections from breast cancer patients using immunocytochemical and clonogenic assay techniques. Blood 82: 2605-2610.

15. Ghossein RA, Bhattacharya S, Rosai J (1999) Molecular detection of micrometastases and circulating tumor cells in solid tumors. Clin Cancer Res 5: 1950-1960.

16. Stott SL, Hsu CH, Tsukrov DI, Yu M, Miyamoto DT, et al. (2010) Isolation of circulating tumor cells using a microvortex-generating herringbone-chip. Proc Natl Acad Sci U S A 107: 18392-18397.

17. Alunni-Fabbroni M, Sandri MT (2010) Circulating tumour cells in clinical practice: Methods of detection and possible characterization. Methods 50: 289-297.

18. Jacob K, Sollier C, Jabado N (2007) Circulating tumor cells: detection, molecul ar profiling and future prospects. Expert Rev Proteomics 4: 741-756.

19. Koyanagi K, O'Day SJ, Boasberg P, Atkins MB, Wang HJ, et al. (2010) Serial monitoring of circulating tumor cells predicts outcome of induction biochemotherapy plus maintenance biotherapy for metastatic melanoma. Clin Cancer Res 16: 2402-2408.

20. Tanaka R, Koyanagi K, Narita N, Kuo C, Hoon DS (2011) Prognostic molecular biomarkers for cutaneous malignant melanoma. J Surg Oncol 104: 438-446.

21. Ghossein RA, Carusone L, Bhattacharya S (2000) Molecular detection of micrometastases and circulating tumor cells in melanoma prostatic and breast carcinomas. In Vivo 14: 237-250.

22. Medic S, Pearce RL, Heenan PJ, Ziman M (2007) Molecular markers of circulating melanoma cells. Pigment Cell Res 20: 80-91.

23. Koyanagi K, O'Day SJ, Gonzalez R, Lewis K, Robinson WA, et al. (2006) Microphthalmia transcription factor as a molecular marker for circulating tumor cell detection in blood of melanoma patients. Clin Cancer Res 12: 1137-1143.

24. Bottoni U, Izzo P, Richetta A, Mannooranparampil TJ, Devirgiliis V, et al. (2003) S100 serum level: a tumour marker for metastatic melanoma. Melanoma Res 13: 427-429.
25. Kumar S, Kishimoto H, Chua HL, Badve S, Miller KD, et al. (2003) Interleukin-1 alpha promotes tumor growth and cachexia in MCF-7 xenograft model of breast cancer. Am J Pathol 163: 2531-2541.

26. Kumar SM, Liu S, Lu H, Zhang H, Zhang PJ, et al. (2012) Acquired cancer stem cell phenotypes through Oct4-mediated dedifferentiation. Oncogene 31 : 4898-4911.

27. Weissenstein U, Schumann A, Reif M, Link S, Toffol-Schmidt UD, et al. (2012) Detection of circulating tumor cells in blood of metastatic breast cancer patients using a combination of cytokeratin and EpCAM antibodies. BMC Cancer 12: 206 .

28. Xu H, Aguilar ZP, Yang L, Kuang M, Duan H, et al. (2011) Antibody conjugated magnetic iron oxide nanoparticles for cancer cell separation in fresh whole blood. Biomaterials 32: 9758-9765.

29. Hirsch LR, Jackson JB, Lee A, Halas NJ, West JL (2003) A whole blood immunoassay using gold nanoshells. Anal Chem 75: 2377-2381.

30. Sharma A, Madhunapantula SV, Robertson GP (2012) Toxicological considerations when creating nanoparticle-based drugs and drug delivery systems. Expert Opin Drug Metab Toxicol 8: 47-69.

31. Clawson GA, Kimchi E, Patrick SD, Xin P, Harouaka R, et al. (2012) Circulating tumor cells in melanoma patients. PLoS One 7: e41052.

32. Mi Y, Li K, Liu Y, Pu KY, Liu B, et al. (2011) Herceptin functionalized polyhedral oligomeric silsesquioxane - conjugated oligomers - silica/iron oxide nanoparticles for tumor cell sorting and detection. Biomaterials 32: 8226-8233.

33. Mejean A, Vona G, Nalpas B, Damotte D, Brousse N, et al. (2000) Detection of circulating prostate derived cells in patients with prostate adenocarcinoma is an independent risk factor for tumor recurrence. J Urol 163: 2022-2029.

34. Vona G, Sabile A, Louha M, Sitruk V, Romana S, et al. (2000) Isolation by size of epithelial tumor cells : a new method for the immunomorphological and molecular characterization of circulatingtumor cells. Am J Pathol 156: 57-63.

35. Krivacic RT, Ladanyi A, Curry DN, Hsieh HB, Kuhn P, et al. (2004) A rare-cell detector for cancer. Proc Natl Acad Sci USA 101: 10501-10504.

36. Gertler R, Rosenberg R, Fuehrer K, Dahm M, Nekarda H, et al. (2003) Detection of circulating tumor cells in blood using an optimized density gradient centrifugation. Recent Results Cancer Res 162: 149-155.

37. Marrinucci D, Bethel K, Bruce RH, Curry DN, Hsieh B, et al. (2007) Case study of the morphologic variation of circulating tumor cells. Hum Pathol 38: 514-519.

38. Koyanagi K, O'Day SJ, Gonzalez R, Lewis K, Robinson WA, Amatruda TT, et al. (2005) Serial monitoring of circulating melanoma cells during neoadjuvant biochemotherapy for stage III melanoma: outcome prediction in a multicenter trial. J Clin Oncol 23: 8057-8064.

39. Takeuchi H, Kuo C, Morton DL, Wang HJ, Hoon DS (2003) Expression of differentiation melanoma-associated antigen genes is associated with favorable disease outcome in advanced-stage melanomas. Cancer Res 63: 441-448.

40. Yu M, Stott S, Toner M, Maheswaran S, Haber DA (2011) Circulating tumor cells: approaches to isolation and characterization. J Cell Biol 192: 373-382.

Citation: Kumar SM (2016) Detection and Molecular Characterization of Circulating Tumor Cells (CTCs) in Patient with Metastatic Melanoma: A Potential Application of Liquid Biopsy. Clin Med Biochem Open Access 2: 119. doi:10.4172/2471-2663.1000119 